\title{
Early constrictive pericarditis and anaemia after Dressler's syndrome and inferior wall myocardial infarction
}

Po Kee Cheung, Mary Lee Myers, J Malcolm O Arnold

\begin{abstract}
Early constrictive pericarditis and anaemia developed in a 52 year old man after he had an inferior wall myocardial infarction complicated by Dressler's syndrome. Total pericardiectomy at the time of coronary artery bypass surgery resulted in complete resolution of signs and symptoms.
\end{abstract}

Constrictive pericarditis after myocardial infarction has been rarely reported. There are three reports of this complication developing six to 12 months after frank haemopericardium had occurred with prophylactic anticoagulant treatment. ${ }^{1-3}$ However, three recent cases occurred in the absence of an overt haemopericardium during the initial post-infarction period and two of these patients did not receive anticoagulants. ${ }^{45}$ The third received an eight week course of anticoagulants for venous thrombosis but this was started four months after his presentation with myocardial infarction and pericarditis. ${ }^{6}$ All six patients had evidence of clinical pericarditis during the acute myocardial infarction and in two patients Dressler's postmyocardial infarction syndrome preceded the development of constrictive pericarditis by six months and 10 months. ${ }^{45}$ Anaemia developed in only one patient and the bone marrow aspirate was consistent with the diagnosis of anaemia of chronic disease. We describe a patient with an inferior wall myocardial infarction who developed constrictive pericarditis and reversible anaemia a month after Dressler's syndrome. Pericardiectomy corrected the constriction and the symptoms resolved.

Department of

Medicine, Victoria

Hospital, University of

Western Ontario,

Lostern Ontario,
Lon, Ontario,

Canada

P K Cheung

J M O Arnold

Department of

Cardiovascular

Surgery, Victoria

Sospital, University of

Western Ontario,

London, Ontario,

Canada

M L Myers

Correspondence to

Dr J Malcolm O Arnold

Division of Cardiology,

Victoria Hospital, Londo

Ontario, Canada N6A 4G5.

\section{Case report}

A 52 year old man was admitted because of acute onset (five hours before) of sharp left sided chest pain which was made worse by breathing and lying down but was improved by sitting forward. He was a long term smoker and had no history of angina or tuberculosis. Physical examination showed a triphasic pericardial rub and bi-basilar crackles but no paradoxical pulse or increase in jugular venous pressure. There were significant $Q$ waves and flattened $T$ waves in the inferior leads of the electrocardiogram with $T$ wave inversion in $I$, aVL, and V4-V6. There was no ST elevation to suggest acute myocardial injury or pericarditis Chest $x$ ray showed mild cardiomegaly and pulmonary congestion and a small loculated pleural effusion. The echocardiogram showed inferoapical akinesis with hypokinesia of the septum and inferior wall. No pericardial effusion was detected. The clinical diagnosis was Dressler's syndrome complicating an inferior myocardial infarction, though it was not possible to determine clearly the timing of the infarction. No serial changes in the electrocardiogram or cardiac enzyme activities were seen. A viral aetiology was considered unlikely because the patient had no preceding history of a recent viral illness and his total and differential white cell counts on admission were normal.

$\mathrm{He}$ was treated with indomethacin $50 \mathrm{mg}$ three times daily for three days then $25 \mathrm{mg}$ three times daily for an additional three days. His pericardial rub and crackles resolved within 48 hours. Neither subcutaneous heparin nor oral warfarin were given. A treadmill exercise test (modified Bruce protocol) was carried out five days after admission and was abnormal with chest pain and ST segment elevation in the inferolateral leads. Cardiac catheterisation showed severe triple vessel coronary artery disease. The right coronary artery was totally occluded in its mid third as was the left anterior descending artery beyond the first major septal perforator. The circumflex artery seemed free of important disease but there was a $75 \%$ proximal stenosis of the intermediate artery. The left ventriculogram showed inferoapical akinesis and a left ventricular ejection fraction of $35 \%$. He was accepted for coronary artery bypass surgery and discharged on once daily atenolol $(100 \mathrm{mg})$ and enteric coated aspirin $(325 \mathrm{mg})$.

He remained free of symptoms for three weeks when he was readmitted because of increasing shortness of breath with anorexia, abdominal and chest discomfort, orthopnoea, and peripheral oedema. Physical examination showed an increased jugular venous pressure of $10 \mathrm{~cm}$ above the sternal angle, pulsus paradoxus (15 $\mathrm{mm} \mathrm{Hg}$ difference), bi-basilar crackles, moderate peripheral oedema, and a mild degree of ascites but no pericardial friction rub. The electrocardiogram had not changed and there were no subsequent changes in serial activities of cardiac enzymes. Because of some clinical evidence suggestive of biventricular failure, he was initially treated with diuretics and intravenous dobutamine and within 24 hours he responded well. An echocardiogram obtained after the start of this treatment showed moderate pericardial effusion and possible early diastolic collapse of the right 
ventricle. Repeat echocardiogram five days later showed resolution of the pericardial effusion and abnormal right ventricular motion. His haemoglobin had dropped from $139 \mathrm{~g} / 1$ at the first admission to $116 \mathrm{~g} / \mathrm{l}$, without any evidence of blood loss or haemolysis. Red cell indices and white cell and platelet counts remained normal.

He was readmitted two weeks later because of increasing orthopnoea with more abdominal distension. Physical examination showed a much increased jugular venous pressure $(12 \mathrm{~cm}$ above the sternal angle), no pulsus paradoxus, a tender liver enlarged to $3 \mathrm{~cm}$ below the right costal margin, moderate ascites, peripheral oedema, and bi-basilar crackles. Again, the electrocardiogram showed no new changes. The echocardiogram showed the previous segmental wall motion abnormalities, no pericardial effusion, but evidence of a paradoxical ventricular septal motion though without appreciable thickening of the pericardium. His haemoglobin concentration had dropped further to $103 \mathrm{~g} / 1$ with normocytic normochromic indices. Reticulocyte count, serum $\mathrm{B12}$, and folate and ferritin concentrations were normal. Antinuclear factor was negative. Bone marrow biopsy showed only non-specific reactive changes. He was treated with diuretics and his clinical state improved somewhat. The decision was made to proceed with surgery.

At operation the anterior pericardium was found to be grossly thickened and firmly adherent to the underlying right ventricle and adjacent portion of the left ventricle as well as to the proximal segments of the aorta and pulmonary artery. There was old haemorrhagic and fibrinous exudate over the anterior aspect of the right atrioventricular groove and right atrium. There was evidence of an earlier large inferior infarction. There was an extensive epicardial inflammatory reaction which made it difficult to identify the coronary vessels. The left internal thoracic artery was used to construct a bypass to the left anterior descending artery and a saphenous vein bypass graft was placed to the intermediate artery. Total pericardiectomy was performed and a significant improvement in right ventricular contractility was noted at the end of the procedure. The right atrial pressure decreased from 25 $\mathrm{mm} \mathrm{Hg}$ before operation to $17 \mathrm{~mm} \mathrm{Hg}$ after operation. By the second postoperative day, it had fallen to $14 \mathrm{~mm} \mathrm{Hg}$. Pathological examination of the excised pericardium showed chronic inflammatory infiltrate throughout its entire thickness (fig) with adherent thrombotic material. One month after operation, the jugular venous pressure was normal and the ascites, peripheral oedema, and hepatomegaly had resolved. A radionuclide blood pool ventriculogram showed normal right ventricular contractility with a right ventricular ejection fraction of $55 \%$. The left ventricle showed inferior segmental wall motion abnormalities and an ejection fraction of $45 \%$. His haemoglobin concentration returned to normal without further need for transfusion after operation. He remained well and symptom free eight months after surgery.

\section{Discussion}

The incidence of early pericarditis during myocardial infarction is reported to vary from $6 \%$ to $23 \%$. $^{7}$ Dressler's post-myocardial infarction syndrome occurs in up to $4 \%$ of patients. ${ }^{8} \mathrm{~A}$ single episode is usually selflimited and responds well to treatment with a non-steroidal anti-inflammatory agent as in this patient. The syndrome may recur though the long term sequelae are not clearly defined. It can be diffuse and may obliterate the pericardial sac, but subsequent development of constrictive
Thickened pericardium showing fibrin $(f)$ and inflammation. (Original magnification $\times 25$.)

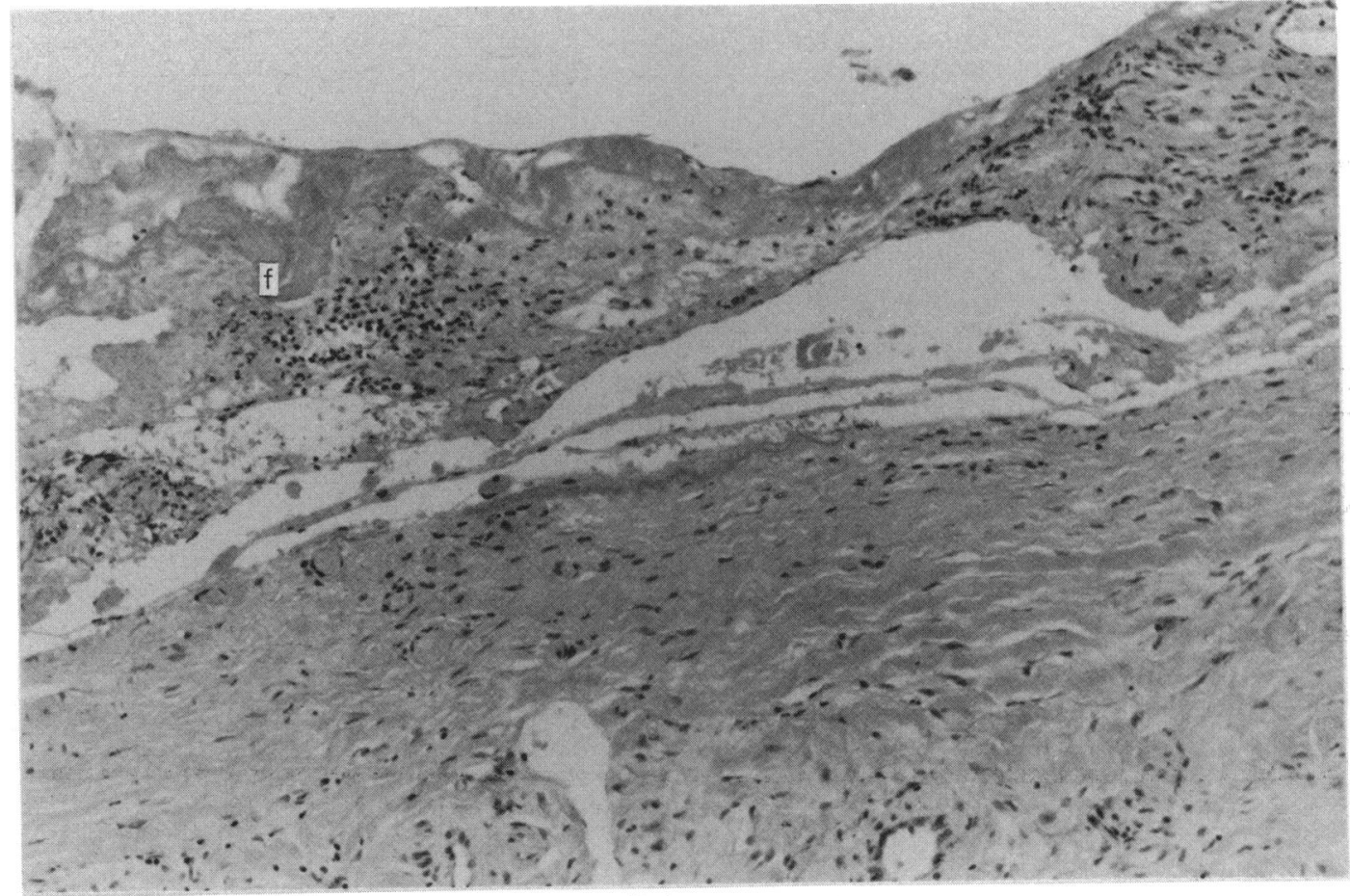


pericarditis has been reported only twice and has occurred six to 10 months after the initial myocardial infarction. ${ }^{45}$ In this patient constrictive pericarditis developed one month after Dressler's syndrome in the absence of prior anticoagulation and in association with anaemia. Some degree of intrapericardial haemorrhage was evident during surgery and histological examination also showed thrombotic material adherent to the pericardium. It is possible that severe pericarditis will cause leakage of blood into the pericardial sac though its contribution to the progression of constrictive pericarditis is not known. All three cases have occurred in men with an inferior wall myocardial infarction. Whether this is a chance association or relates to an anatomical factor, such as increased adherence of the inferior pericardium to the diaphragm or myocardium, is not known. All showed an excellent response to pericardiectomy.

A few cases of protracted Dressler's syndrome have been associated with anaemia, but the aetiology was not clear because bone marrow studies were not performed. ${ }^{9}$ Our patient showed the development of early reversible anaemia and constrictive pericarditis in Dressler's syndrome similar to that reported by Kanawaty et al. ${ }^{5}$ Bone marrow biopsy in both cases showed non-specific changes. It is possible that myocardial injury initiated an immune reaction leading to both pericarditis and anaemia. It is interesting to speculate that the early development of anaemia may be a marker for a more severe immune response. Recent evidence suggests that inflammatory mediators, such as interleukin 1 , released in severe inflammatory conditions may decrease the erythroblast response to erythropoietin and cause anaemia which may respond to corticosteroids. $^{10} \mathrm{~A}$ recent retrospective analysis of a related condition, postpericardiotomy syn- drome, concluded that steroids, non-steroidal anti-inflammatory drugs, or the combination did not seem to prevent the future development of constrictive pericarditis. ${ }^{11}$ A prospective controlled trial in tuberculous pericarditis suggested that adjunctive treatment with prenisolone improves clinical symptoms and survival as well as reducing pericardiocentesis and possibly pericardiectomy. ${ }^{12}$ Further studies may be needed to resolve this issue in the subgroup of patients with Dressler's syndrome and anaemia.

Dr J M O Arnold is the recipient of a Career Health Scientist Award from the Pharmaceutical Manufacturers Association of Canada. We thank Dr M Troster for the pathological analysis Canada. We thank
of the pericardium.

1 Laszlo MH. Constrictive pericarditis as sequel to hemopericardium: report of a case following anticoagulan therapy. Ann Intern Med 1957;46:403-13.

2 Beaufils PH, Bardet J, Temkine J, Masquet $\mathrm{CH}$, Sebastien PH, Zelasko Y. Syndrome de Dressler: pericardite constrictive post-infarctus opérée avec succès. Arch Mal Coeur 1975;68:651-6.

3 Karim AH, Salomon J. Constrictive pericarditis after myocardial infarction: sequela of anticoagulant-induced hemopericardium. Am J Med 1985;79:389-90

4 Goldhaber SZ, Lorell BH, Green LH. Constrictive pericarditis, a case requiring pericardiectomy following Dressler's post myocardial infarcti

5 Kanawaty DS, Burggraf GW, Abdollah H. Constrictive pericarditis and anaemia post myocardial infarction. Can pericarditis and anaemia post

6 Ramsdale DR, Epstein EJ, Coulshed N. Constrictive pericarditis after myocardial infarction. Br Heart J 1986; 56:476-8

7 Toole JC, Silverman ME. Pericarditis of acute myocardial infarction. Chest 1975;67:647-53.

8 Davidson C, Oliver MD, Robertson RF. Post-myocardia infarction syndrome. BMJ 1961;ii:535-9.

9 Glasser M. Post-myocardial infarction syndrome with anaemia. Conn Med J 1970;34:413-4.

10 Schooley JC, Kullgren B, Allison AC. Inhibition by interleukin-I of the action of erythropoietin on erythroid precursors and its possible role in the pathogenesis of precursors and its possible role in the pathogen

11 Cimino JJ, Kogan AD. Constrictive pericarditis after cardiac surgery: report of three cases and review of the literature. surgery: report of three cases and

12 Strang JIG, Kakaza HH, Gibson DG, et al. Controlled trial of complete open surgical drainage and of prednisolone treatment of tuberculous pericardial effusion in Transkei Lancet 1988;ii:759-64. 